\title{
Corrigendum: Reduced Mu Power in Response to Unusual Actions Is Context-Dependent in 1-Year-Olds
}

\author{
Miriam Langeloh ${ }^{1,2 *}$, David Buttelmann ${ }^{3}$, Daniel Matthes ${ }^{1}$, Susanne Grassmann ${ }^{4}$, \\ Sabina Pauen ${ }^{2}$ and Stefanie Hoehl ${ }^{1,5}$ \\ ${ }^{1}$ Max Planck Institute for Human Cognitive and Brain Sciences, Leipzig, Germany, ${ }^{2}$ Department of Psychology, Heidelberg \\ University, Heidelberg, Germany, ${ }^{3}$ Department of Psychology, University of Bern, Bern, Switzerland, ${ }^{4}$ Institute of Educational \\ Research and Development, University of Applied Sciences and Arts Northwestern Switzerland, Windisch, Switzerland, \\ ${ }^{5}$ Faculty of Psychology, University of Vienna, Vienna, Austria
}

Keywords: EEG, infants, action perception, action understanding, mu frequency, mirror neuron system

\section{A Corrigendum on}

Reduced Mu Power in Response to Unusual Actions Is Context-Dependent in 1-Year-Olds

OPEN ACCESS

Edited by: Markus Paulus,

Ludwig Maximilian University of

Munich, Germany

Reviewed by:

Janny Christina Stapel,

Uppsala University, Sweden

${ }^{*}$ Correspondence:

Miriam Langeloh

langeloh@cbs.mpg.de

Specialty section

This article was submitted to Developmental Psychology, a section of the journal

Frontiers in Psychology

Received: 01 December 2018 Accepted: 01 February 2019

Published: 20 February 2019

Citation:

Langeloh M, Buttelmann D, Matthes D, Grassmann S, Pauen S and Hoehl S (2019) Corrigendum: Reduced Mu Power in Response to

Unusual Actions is

Context-Dependent in 1-Year-Olds.

Front. Psychol. 10:316.

doi: 10.3389/fpsyg.2019.00316 by Langeloh, M., Buttelmann, D., Matthes, D., Grassmann, S., Pauen, S., and Hoehl, S. (2018). Front. Psychol. 9:36. doi: 10.3389/fpsyg.2018.00036

In the original article, there were mistakes in Figures $\mathbf{2 - 5}$ as published. We analyzed the artifactfree data segments in Fieldtrip (Oostenveld et al., 2011) using the "ft_freqanalysis" function. We configured this function to compute power, however, stated erroneously in the original text that we computed the "power spectral density (PSD)." Consequently, we labeled the y-axis units according to PSD but not power.

The y-axis unit in Figure 3 was corrected to " $\mu V^{2}$ ", additionally, the scaling used in Figures 2, 4 and 5 was a natural logarithm instead of a common logarithm. The scaling has now been adjusted to the common logarithm and the y-axis unit has been adjusted to " $\mathrm{dB}$ " accordingly. The corrected Figures 2-5 appear below.

A correction has also been made to the Materials and Methods, EEG Recording and Analyses, Frequency Domain Analysis, Paragraph one:

"Artifact-free data segments were submitted to fast Fourier transformations (FFTs). For each segmented test frame (hand or head touch), the power was computed from 0 to 1,500 ms relative to the onset of the related stimulus using a Hanning-tapered window of the same length (by applying the ' $\mathrm{ft}$ freqanalysis' function with 'mtmfft' method as implemented in Fieldtrip). Power estimates were calculated for frequencies $\left(\frac{2}{3} \mathrm{~Hz}\right.$ bins) between 0 and $124.667 \mathrm{~Hz}$. Grand averages of the FFTs were computed for both hand- and head-action outcomes in the hands-free and hand-restrained condition."

Additionally, there was a mistake in the legend for Figure 2 as published. The legend has been rewritten to provide a better understanding of the figure content. The correct legend appears below.

"Figure 2. Individual power spectra across an average for hand- and head-touch actions across an average of frontal and central electrodes (F3, F4, C3, C4) for (A) hands-free and (B) hands-restrained condition."

The authors apologize for these errors and state that they do not change the scientific conclusions of the article in any way. The original article has been updated. 


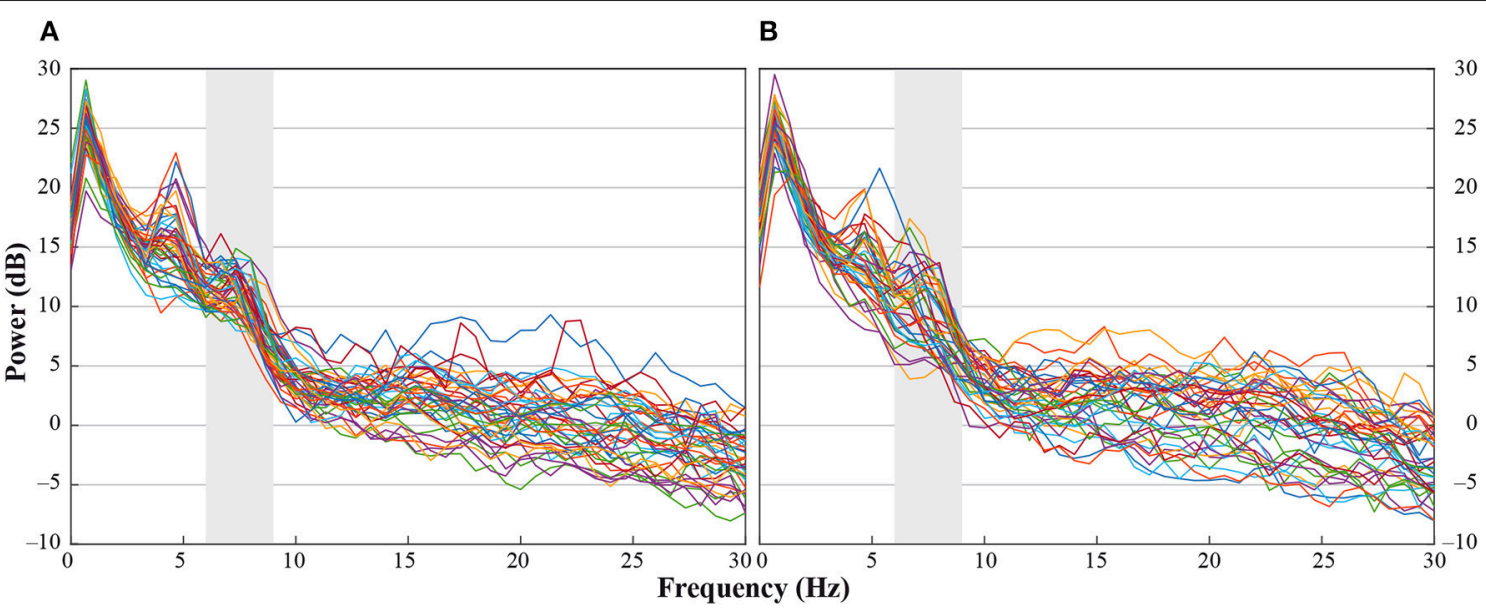

FIGURE 2 | Individual power spectra across an average for hand- and head-touch actions across an average of frontal and central electrodes (F3, F4, C3, C4) for (A) hands-free and (B) hands-restrained condition.

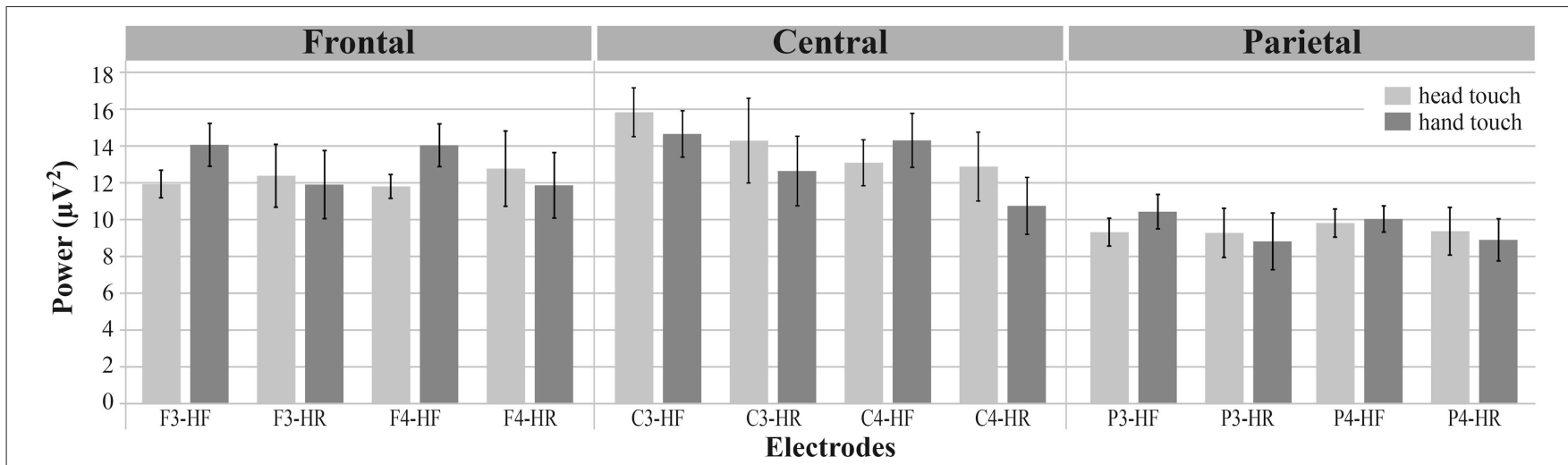

FIGURE 3 | Grand average EEG power across mu frequency band (6-8 Hz) for electrodes of interest (F3, F4, C3, C4, P3, P4) in response to hand touch (dark gray) and head touch (light gray) for both hands-free (HF) and hands-restrained (HF) condition. Error bars represent standard errors of the mean.

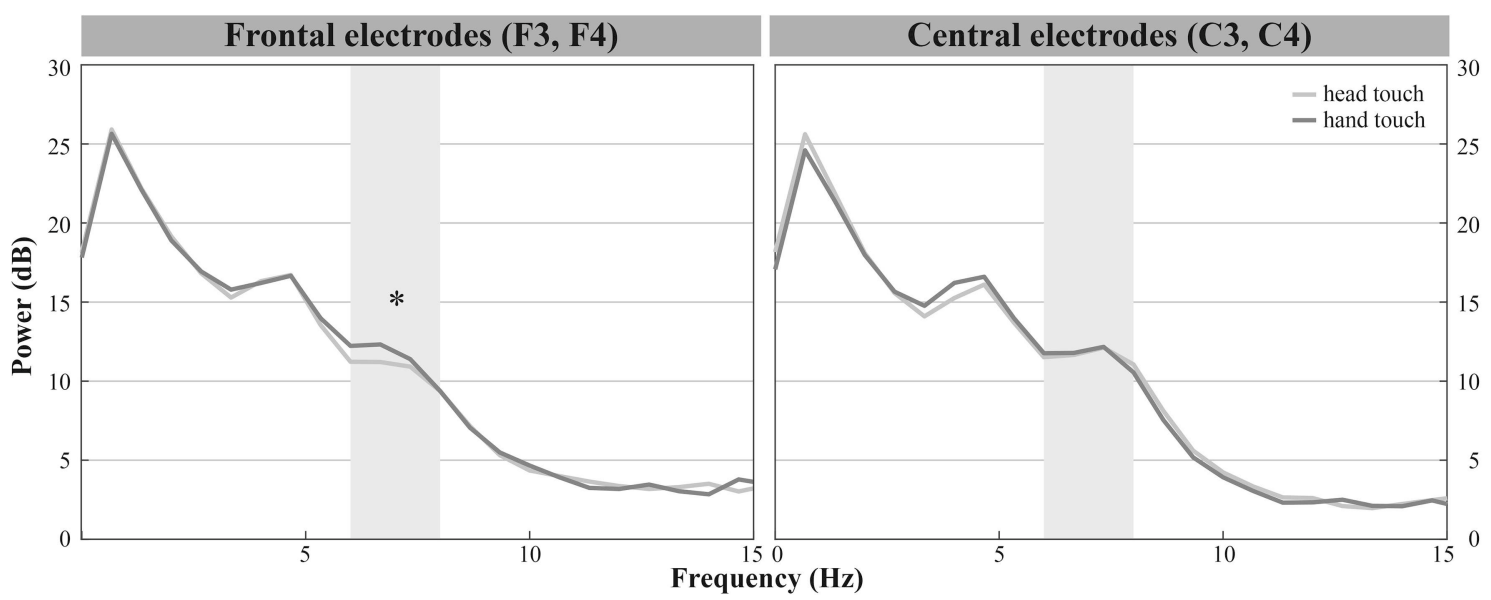

FIGURE 4 | Grand average EEG mu power for hand touch (dark gray) and head touch (light gray) for an average of frontal electrodes (F3, F4) and for an average of central electrodes $(\mathrm{C} 3, \mathrm{C} 4)$ in the hands-free condition. Asterisks depict significant differences with $p<0.05$. 


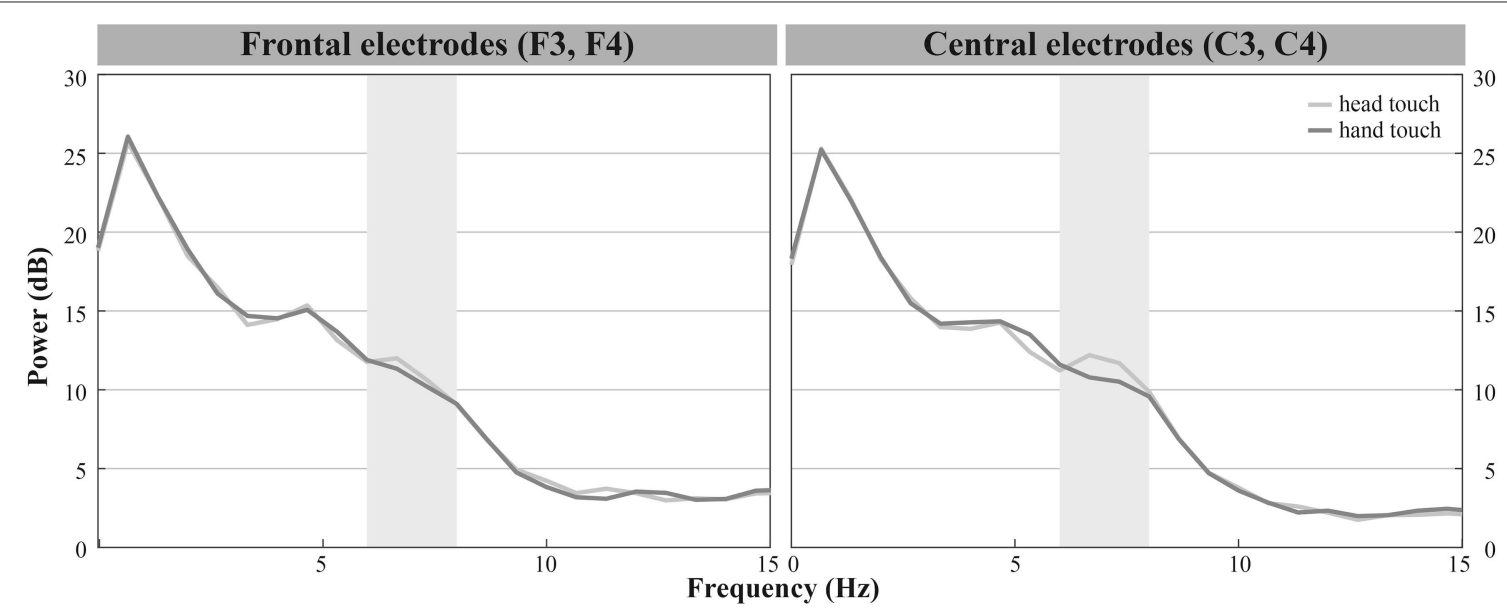

FIGURE 5 | Grand average EEG mu power for hand touch (dark gray) and head touch (light gray) for an average of frontal electrodes (F3, F4) and for an average of central electrodes $(\mathrm{C} 3, \mathrm{C} 4)$ in the hands-restrained condition.

\section{REFERENCES}

Oostenveld, R., Fries, P., Maris, E., and Schoffelen, J. M. (2011). FieldTrip: open source software for advanced analysis of MEG, EEG, and invasive electrophysiological data. Comput. Intell. Neurosci. 2011:156869. doi: $10.1155 / 2011 / 156869$
Copyright $\odot 2019$ Langeloh, Buttelmann, Matthes, Grassmann, Pauen and Hoehl. This is an open-access article distributed under the terms of the Creative Commons Attribution License (CC BY). The use, distribution or reproduction in other forums is permitted, provided the original author(s) and the copyright owner(s) are credited and that the original publication in this journal is cited, in accordance with accepted academic practice. No use, distribution or reproduction is permitted which does not comply with these terms. 